\title{
Theory behind formation of Neutrino and procedure of calculating mass of Neutrino under various circumstances
}

\author{
Mr. Gaurav S. Dhage \\ Former student of Mechanical Engineering at Pune University, \\ 10 Aaisaheb, kale Nagar-2, Aanandwalli, Nashik, India \\ gauravdhage2013@gmail.com
}

\begin{abstract}
Study of neutrino is very important aspect of particle physics. Neutrino has unique characteristics like zero charge and negligible mass compared to other subatomic particles which makes extremely difficult to analyse and to study behaviour of Neutrino; through experimental studies. So Neutrino is still unknown territory for humankind. Understanding how neutrinos operate is essential for next steps in new physics. As it is difficult to study them experimentally; I am using theoretical approach in this research paper to study Neutrino. In this research paper I have expressed my thoughts and proposed hypothesis. Based on hypothesis I have derived formula for calculation of Neutrino's mass. In this paper I have also observed some properties Neutrino based on my hypothesis.
\end{abstract}

\section{KEYWORDS}

Neutrino

\section{ACADEMIC DISCIPLINE AND SUBJECT}

Science

\section{SUBJECT CLASSIFICATION}

Physics

\section{TYPE (METHOD/APPROACH)}

Theorotical Approach, Analysis

\section{INTRODUCTION}

Mass of Neutrino and its origin are still one of the biggest unsolved problems in physics. In this paper I am trying to solve these problems. Aim of this research paper is to study and understand theory of Neutrino and find out unknown properties of Neutrino. In $\beta$ particle decay phenomenon parent nuclide converts into daughter nuclide and $\beta$ particle, Neutrino emitted. But origin of Neutrino is unknown. In this Research paper I will try to solve this problem theoretically.

\section{DHAGE'S HYPOTHESIS}

Energy $(E 1)$ equal to difference between the observed energy for $\beta$ particle and maximum energy of continuous $\beta$ spectrum; is distributed in following two phenomena:

1) Part of above energy, say E2 is converted to mass or matter of Neutrino and thus Neutrino is created.

2) Remaining energy, say $E 3$ is carried by Neutrino in form kinetic energy. i.e. $E 1=E 2+E 3$

\section{Calculation of mass of Neutrino in various circumstances:}

$E 1=E 2+E 3$

Where,

$E 1=$ difference between the observed energy for $\beta$ particle and maximum energy of continuous $\beta$ spectrum in joule;

$E 2=m c^{2}$ in joule;

$E 3=$ kinetic energy of Neutrino $=\left(m c^{2} / \sqrt{ }\left(1-\left(\frac{v}{c}\right)^{2}\right)\right)-m c^{2}$ in joule; 
$\mathrm{v}=$ velocity achieved by Neutrino in $\mathrm{m} / \mathrm{sec}$;

$\mathrm{c}=$ velocity of light in vacuum in $\mathrm{m} / \mathrm{sec}$

Hence,

$E 1=m c^{2}+\left(m c^{2} / \sqrt{ }\left(1-\left(\frac{v}{c}\right)^{2}\right)\right)-m c^{2}$

$E 1=\left(m c^{2} / \sqrt{ }\left(1-\left(\frac{v}{c}\right)^{2}\right)\right)$

$\mathrm{m}=\left(E 1^{*}\left(\sqrt{ }\left(1-\left(\frac{v}{c}\right)^{2}\right)\right)\right) / \epsilon^{2}$

For a particular case

$E 1=1 \mathrm{eV}$

$(\mathrm{v} / \mathrm{c})=1-10^{\wedge}-7=0.9999999(\mathrm{MINOS} 2015) ;$

So mass of neutrino is $=8.0142^{*} 10^{\wedge}-40 \mathrm{~kg}$

Thus by knowing value of $E 1$ and velocity of Neutrino we can find out mass of that Neutrino which formed in $\beta$ particle decay phenomenon.

\section{DISCUSSIONS}

1) Neutrino created in $\beta$ particle decay phenomenon by conversion of energy into matter.

2) Mass of Neutrino is dependent on energy $=E 1$

\section{ACKNOWLEDGMENTS}

My thanks to my parents

\section{REFERENCES}

1) Einstein's theory of special relativity.

2) MINOS 2015

\section{AUTHOR'S BIOGRAPHY WITH PHOTO}

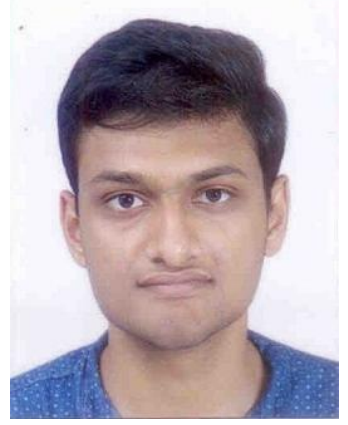

Mr. Gaurav S. Dhage is born in India in 1995 and Aumnus of Mechanical Engineering at Pune University in 2016.

10 Aaisaheb, kale Nagar-2, Aanandwalli, Gangapur Road,

Nashik, Indian State of Maharashtra, India

Pin code- 422013

Mobile number- +919922773706

Email Id-gauravdhage2013@gmail.com, gauravdhage22@yahoo.in

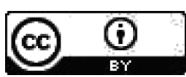

This work is licensed under a Creative Commons Attribution 4.0 International License.

DOI : 10.24297/jap.v13i8.6333 